\title{
Que pensent les élus municipaux québécois des missions des bibliothèques publiques?
}

Résultats d'une étude de cas

What do Elected Municipal Officials Think of the Mission of

Public Libraries?

\section{¿Qué opinan los funcionarios municipales electos} quebequenses de las misiones de las bibliotecas públicas?

\section{Resultados de un estudio de caso}

\section{Dominique Gazo}

Volume 56, numéro 1, janvier-mars 2010

URI : https://id.erudit.org/iderudit/1029168ar

DOI : https://doi.org/10.7202/1029168ar

\section{Aller au sommaire du numéro}

\section{Éditeur(s)}

Association pour l'avancement des sciences et des techniques de la documentation (ASTED)

\section{ISSN}

0315-2340 (imprimé)

2291-8949 (numérique)

\section{Découvrir la revue}

\section{Citer cet article}

Gazo, D. (2010). Que pensent les élus municipaux québécois des missions des bibliothèques publiques ? Résultats d'une étude de cas. Documentation et bibliothèques, 56(1), 5-13. https://doi.org/10.7202/1029168ar
Résumé de l'article

Cet article présente les résultats d'une étude menée dans le cadre d'un projet de recherche doctoral. Douze élus municipaux québécois ont été interrogés sur les missions des bibliothèques publiques. Ils s'expriment en tant qu'élu, individu ou usager pour évoquer une bibliothèque qui paraît passive et traditionnelle. Ces élus ne parviennent pas à imaginer une bibliothèque autre que celle qu'ils connaissent présentement et qui les satisfait généralement. Malgré ses limites concernant surtout les valeurs, les publics, le personnel et la variété des services offerts, le discours des élus ressemble étrangement au discours du public et converge avec celui des professionnels.
Tous droits réservés $@$ Association pour l'avancement des sciences et des techniques de la documentation (ASTED), 2010
Ce document est protégé par la loi sur le droit d'auteur. L'utilisation des services d'Érudit (y compris la reproduction) est assujettie à sa politique d'utilisation que vous pouvez consulter en ligne.

https://apropos.erudit.org/fr/usagers/politique-dutilisation/ 


\section{RÉSUMÉ | ABSTRACTS | RESUMEN}

Cet article présente les résultats d'une étude menée dans le cadre d'un projet de recherche doctoral. Douze élus municipaux québécois ont été interrogés sur les missions des bibliothèques publiques. Ils s'expriment en tant qu'élu, individu ou usager pour évoquer une bibliothèque qui paraît passive et traditionnelle. Ces élus ne parviennent pas à imaginer une bibliothèque autre que celle qu'ils connaissent présentement et qui les satisfait généralement. Malgré ses limites concernant surtout les valeurs, les publics, le personnel et la variété des services offerts, le discours des élus ressemble étrangement au discours du public et converge avec celui des professionnels.

What do Elected Municipal Officials Think of the Mission of Public Libraries?

This article presents the results of a study undertaken as part of a doctoral research project. Twelve elected Québec municipal officials were surveyed regarding their perception of the mission of public libraries. Their perception, either as elected officials, individuals or users, is that libraries are passive and traditional. These elected officials cannot imagine a library other than the one they know and with which they are generally satisfied. In spite of the limits of public libraries regarding values, the public, the personnel and the range of services offered, the position of the elected officials is quite similar to that of the public and converges with that of the professionals.

$¿ Q u e ́$ opinan los funcionarios municipales electos quebequenses de las misiones de las bibliotecas públicas? Resultados de un estudio de caso

Este artículo presenta los resultados de un estudio realizado en el marco de un proyecto de investigación doctoral. Se solicitó la opinión de doce funcionarios municipales electos quebequenses acerca de las misiones de las bibliotecas públicas. Estos se expresaron como funcionarios electos, como personas o como usuarios, evocando una biblioteca que puede parecer pasiva y tradicional. Para estos funcionarios electos, es difícil imaginar una biblioteca distinta de la que conocen en la actualidad y que los satisface plenamente. Dentro de los límites que incluyen los valores, los distintos públicos, el personal y la variedad de servicios ofrecidos, el discurso de los funcionarios electos se parece extrañamente al discurso del público en general y coincide con el de los profesionales.

\section{Introduction}

$\mathrm{D}$ EPUIS PLUSIEURS DÉCENNIES, les bibliothèques publiques sont dans la tourmente de la société de l'information. Elles ont atteint un point au-delà duquel la philosophie qui prévalait jusqu'alors est déstabilisée ; on parle de changement de paradigme (McKee, 1987). On distingue désormais le paradigme traditionnel, caractérisé par une vision communautaire et sociétale des missions des bibliothèques publiques, du paradigme postmoderne qui se caractérise par une vision individualiste de ces missions (Muddiman, 1990). Dans ce débat, le seul point d'accord entre les deux paradigmes est le bénéfice à tirer d'un questionnement sur les missions des bibliothèques publiques d'aujourd'hui. Le besoin de redéfinir les missions des bibliothèques publiques pour conforter leur légitimité est reconnu par les chercheurs et ressenti par les responsables des bibliothèques. Ces derniers craignent que le doute ne soit jeté sur les missions des bibliothèques publiques, sur leur utilité même (Gomez \& Hernandez, 1998 ; Kerslake \& Kinnell, 1998 ; Rubin, 2004) et sur leur légitimité (KannChristensen \& Pors, 2004). De plus, rares sont les pays qui ont défini les missions des bibliothèques publiques dans des textes législatifs, comme l'ont fait le Danemark ou la Belgique (Poulain \& Darrobers, 1992). L'UNESCO (1994) ainsi que plusieurs associations professionnelles telles l'IFLA (2001), l'ALA (McClure et al., 1987) et l'ASTED (1996) ont traité des missions des bibliothèques publiques, mais sans que leurs propos aient force de loi. Les textes de référence dans le domaine des bibliothèques publiques sont pluriels (Tableau 1) et ambigus (Bouthillier, 1996). Ceci n'aide pas la prise de décision en ce qui concerne la définition des missions des bibliothèques publiques. Or, une mission peu ou mal définie est source de tension, d'instabilité chez les professionnels (March \& Olsen, 1976) et d'interprétations multiples chez les élus dans le cas d'institutions publiques (Bouthillier, 1995 et 1997). Les responsables des bibliothèques publiques québécoises se plaignent justement de l'incompréhension et du désintérêt des élus vis-à-vis les bibliothèques publiques (Baillargeon, 


\section{Tableau 1}

Les missions des bibliothèques publiques dans les textes officiels.

\begin{tabular}{|l|l|l|l|}
\hline UNESCO (1994) & IFLA (2000) & ALA (1987) & ASTED (1996) \\
\hline Information & Éducation & Centre d'activités communautaire & Accès à l'information \\
Alphabétisation & Information & Centre d'information & Développement de la culture \\
Éducation & Développement & communautaire & Diffusion de la culture québécoise \\
Culture & personnel & Centre de support éducatif & Soutien aux activités éducatives, \\
& Enfants et jeunes & Centre indépendant & fiques, récréatives et culturelles \\
& Développement culturel & d'apprentissage & \\
& & Bibliothèque populaire & Porte d'apprentissage aux enfants \\
& & d'âge préscolaire & \\
& & Bibliothèque de référence & \\
& & Centre de recherche & \\
\hline
\end{tabular}

2002 ; Lefebvre-Roux, 1993), alors que ces mêmes élus ont le sort des bibliothèques publiques entre leurs mains (Lajeunesse, 2005).

\section{L'étude}

Devant cette problématique, une étude sur les missions des bibliothèques publiques, telles quévoquées par les élus municipaux québécois, a été menée dans le cadre d'une recherche menant à l'obtention d'un doctorat, sous la direction de Réjean Savard, professeur titulaire à l'École de Bibliothéconomie et des Sciences de l'Information (EBSI) de l'Université de Montréal'. L'objectif principal de cette recherche était de comprendre, via les discours, le point de vue des élus municipaux québécois sur les missions des bibliothèques publiques, pour les comparer ensuite avec les pratiques dans ces bibliothèques. Pour atteindre cet objectif, une étude de cas multiples a été réalisée. Les statistiques pour l'année 2006 des bibliothèques publiques autonomes ont été utilisées. Lors de létablissement de notre échantillon, les villes situées à plus de $150 \mathrm{~km}$ de l'Université de Montréal ont été retirées de la liste des 106 villes dotées d'une bibliothèque publique autonome, pour des raisons logistiques. Les 64 villes restantes ont été réparties en 6 ensembles en fonction de leur taille (grande ville, ville moyenne, petite ville) et de l'allocation municipale octroyée à la bibliothèque (égale ou supérieure à la moyenne provinciale, inférieure à la moyenne provinciale). Dans chacun de ces 6 ensembles, deux cas ont ensuite été choisis aléatoirement. L'échantillon était donc constitué de 12 cas. Pour chacun de ces cas, des entrevues ont été menées auprès des élus municipaux en charge du dossier "bibliothèque ", que ce soit via un conseil d'administration, un comité municipal ou une commission municipale. Ces entrevues, ainsi que les politiques culturelles municipales, ont fait lobjet d'une

1. Le texte intégral de la thèse est disponible à l'adresse suivante : $<$ https://papyrus.bib.umontreal.ca/jspui/bitstream/1866/3030/1/ Gazo-D-missions-bibliotheques-publiques-par-elus.pdf $>$. analyse de discours afin d'en extraire les éléments thématiques et contextuels les plus significatifs. Des entrevues ont également été menées auprès des responsables des bibliothèques dans les 12 villes constituant notre échantillon. La documentation concernant ces bibliothèques a été analysée. Une analyse de contenu a permis d'extraire des entrevues et des documents ainsi que des renseignements contextuels et factuels intéressants.

Afin de respecter la confidentialité des données et déviter que les villes ou les individus ne soient reconnus, seuls les résultats globaux sont présentés ici. Mais auparavant, une présentation des 12 élus ayant participé à l'étude s'impose.

\section{Les douze élus participants}

Les 12 municipalités de notre échantillon se situent dans les régions administratives du Centre du Québec, de l'Estrie, de Lanaudière, des Laurentides, de la Mauricie et de la Montérégie.

Le hasard a fait que léchantillon était constitué en égale proportion d'élus masculins et féminins. Les élus proviennent d'horizons professionnels très variés et un tiers d'entre eux détient un diplôme universitaire. Dix élus nen sont pas à leur premier mandat en politique municipale. Onze élus sur les douze rencontrés ont plus de 45 ans et trois élus seulement ont fréquenté une bibliothèque publique dans leur enfance. Dix élus municipaux sur douze sont présentement inscrits à la bibliothèque ; les deux autres disent préférer acheter des livres. Signalons que les trois élus qui ont fréquenté une bibliothèque publique étant plus jeunes sont tous des usagers aujourd'hui. Mais la "pesanteur sociologique " qui touche, d'après Baillargeon (2002, p. 39), cette génération de Québécois qui nont pas connu de bibliothèque publique dans leur enfance, n'est peut-être pas si déterminante qu'il le craignait.

Les élus que nous avons rencontrés se sont montrés plus ou moins intéressés par le domaine des bibliothèques publiques. Certains d'entre eux (un tiers de léchan- 
tillon) avouent que leur intérêt est né au fur et à mesure que s'intensifiait leur implication dans le domaine. Les sollicitations des citoyens piquent la curiosité et suscitent l'intérêt de leurs élus. D’autres, un tiers de l'échantillon, ne croient pas que leur élection ait modifié leur point de vue quant aux bibliothèques publiques ; au mieux, ils se disent "plus sensibilisés" et "plus au courant" de ce qui se fait en bibliothèques. Pour les trois élus qui ont fréquenté une bibliothèque publique dans leur enfance et pour l'élu d'une ville moyenne (un enseignant), l'intérêt pour la bibliothèque était présent bien avant leur élection. Cinq élus se disent particulièrement surpris par l'achalandage et les différentes utilisations des bibliothèques. Dans les entrevues que nous avons menées, l'intérêt des élus est donc palpable. Qu'ils apprécient les services de la bibliothèque depuis leur jeunesse ou depuis leur élection, cet intérêt existe et il peut être exploité par les professionnels (Baillargeon, 2002 ; Lefebvre-Roux, 1993).

Plusieurs élus se sont fait une autre image de la bibliothèque après leur élection. Ceux-ci considèrent désormais la bibliothèque du point de vue de leur rôle d'élu municipal, de leurs propres convictions et des utilisations réelles ou supposées de la bibliothèque.

\section{Quand l'élu s'exprime en tant qu'élu}

Notre recherche a montré que les élus s'exprimaient en tant que responsables du bien-être des citoyens. De ce point de vue, les élus sont particulièrement sensibles à la satisfaction des citoyens, à l'image de leur ville et à la paix sociale, ainsi qu'à la centralité, laccessibilité et la qualité de la bibliothèque.

\section{La satisfaction des citoyens}

La plupart des élus sont particulièrement sensibles à la fréquentation de la bibliothèque (dix élus sur douze) : plus nombreux sont les usagers, plus nombreux sont les électeurs satisfaits. Les élus y trouvent donc leur compte en vue d'une réélection possible. Mais alors que le nombre de visiteurs est un résultat tangible de la bibliothèque, les services qui y sont offerts sont par contre souvent invisibles.

\section{Une ville attrayante et comparable aux autres}

Trois élus mettent de l'avant l'attrait que constitue la bibliothèque pour leur ville. La bibliothèque est vue comme un atout qui rapporte quelque chose à la ville. La bibliothèque est alors un investissement et non un service. Cette vision de la bibliothèque comme vecteur d'image pour la ville est cependant loin d'être partagée par tous (Grandmont, 2004).

La moitié des élus des petites municipalités sont curieux de savoir comment cela se passe dans d'autres

\section{Les élus sont particulièrement sensibles à la satisfaction des citoyens, à l'image de leur ville et à la paix sociale, ainsi qu'à la centralité, l'accessibilité et la qualité de la bibliothèque.}

00000000000000000000000000000000000000000

villes de même taille. Ils comparent leur ville, leur bibliothèque, aux autres. Bien souvent, lélu a une vision juste de sa municipalité mais ne sait pas bien ce qui se passe ailleurs.

\section{La paix sociale}

Pour trois élus de grandes villes ou de villes moyennes, la bibliothèque joue un rôle dans la lutte au décrochage scolaire et à la criminalité. Pour ce faire, inutile de créer de nouveaux services. Simplement, «un jeune qui est à la bibliothèque, ne traîne pas dans les rues " à faire du grabuge. Ou encore, "un jeune qui lit a moins de chance de verser dans la marginalité ». Pour ces élus, la bibliothèque a donc un rôle à jouer dans la promotion de la paix sociale; elle peut en même temps permettre lascension de l'individu dans la société.

\section{La centralité}

La bibliothèque est décrite comme "carrefour", comme « cœur de la ville ». Dans le premier cas, il est question de centralité physique et d'accessibilité. Dans le second cas, il s'agit de centralité des fonctions vitales. La bibliothèque apparait donc comme un lieu central physiquement et fonctionnellement.

\section{L'accessibilité}

Pour les élus que nous avons rencontrés, l'accessibilité se décline en termes d'heures d'ouverture et de gratuité des services. Neuf bibliothèques sur douze offrent un abonnement gratuit pour le prêt de tous les types de documents; les élus de ces neuf bibliothèques se sont montrés particulièrement fiers de ces conditions. Dans le discours des trois élus des villes résistant à la gratuité complète de l'abonnement, l'accessibilité financière est pourtant présentée comme une nécessité. Cet écart entre le discours et les pratiques apparait dans les villes dont l'allocation municipale par habitant octroyée à la bibliothèque est inférieure à la moyenne provinciale. La problématique de la gratuité continue donc de se poser, même si le discours affiché par les élus municipaux est très prometteur. Pour accroître l'accessibilité des bibliothèques, l'augmentation des heures douverture est quelquefois préconisée notamment par les élus des petites villes et des villes moyennes, sans doute parce qu'il reste du chemin à faire en ce domaine. Dans d'autres 


\section{Tableau 2}

La direction des bibliothèques de l'échantillon.

\begin{tabular}{|c|c|c|c|c|c|}
\hline $\begin{array}{l}\text { DIRECTION DE LA } \\
\text { BIBLIOTHÈQUE }\end{array}$ & $\begin{array}{c}\text { GRANDES } \\
\text { VILLES } \\
(\mathbf{N}=4)\end{array}$ & $\begin{array}{l}\text { VILLES } \\
\text { MOYENNES } \\
(\mathrm{N}=4)\end{array}$ & $\begin{array}{c}\text { Petites } \\
\text { VILles } \\
(\mathrm{N}=4)\end{array}$ & $\begin{array}{c}\text { ALLOCATION PAR } \\
\text { HABITANT } \\
<\text { MOYENNE }(\mathrm{N}=6)\end{array}$ & $\begin{array}{c}\text { ALLOCATION PAR } \\
\text { HABITANT } \\
\geq \text { MOYENNE }(N=6)\end{array}$ \\
\hline Bibliothécaire & 3 villes & 4 villes & & 3 villes & 4 villes \\
\hline Technicien & & & 3 villes & 2 villes & 1 ville \\
\hline Autre & 1 ville & & 1 ville & 1 ville & 1 ville \\
\hline
\end{tabular}

cas, les élus sont récalcitrants face à léventualité délargir les heures douverture, probablement parce quouvrir plus longtemps la bibliothèque signifie augmenter les heures de travail des employés et/ou des bénévoles.

Dans la littérature professionnelle, on parle dégalité ou d'équité d'accès, une valeur primordiale pour les professionnels (Koehler, Hurych, Dole \& Wall, 2000 ; Smith, 2008). De même, les élus rencontrés font montre d'un souci certain pour l'accessibilité de la bibliothèque, mais cette accessibilité ne se décline pas de la même manière selon les contextes. L'accessibilité de la bibliothèque pose donc problème dans la pratique, même si c'est une valeur récurrente dans le discours des élus municipaux québécois. Certains élus prônent laccessibilité certes, mais à condition quelle nait pas un coût.

\section{La qualité du service}

Les animations sont le principal vecteur de qualité du service selon la plupart des élus municipaux que nous avons rencontrés (neuf élus sur douze). Toutefois, leur discours est loin dêtre représentatif de ce qui se fait réellement dans les bibliothèques. Le plus souvent, les élus ne connaissent des animations que les activités destinées aux écoles ou les activités qui ont lieu à la bibliothèque, alors que les bibliothèques offrent déjà ou ont en projet toute une panoplie d'activités destinées à tous les publics, dans et hors les murs. De même, le public méconnaît les services offerts par les bibliothèques publiques (OCLC, 2005). Aux yeux des professionnels, le service à la clientèle, la référence et le conseil de lecture sont trois des plus importants services offerts aux usagers des bibliothèques (Smith, 2008). Or, ceux-ci n'apparaissent aucunement dans le discours des élus. Pourtant, un tiers des élus rencontrés s'est dit "plus au courant " de ce qui se fait en bibliothèque, mais en parallèle cinq élus sur douze mettent en cause le marketing des bibliothèques. Les professionnels ont donc intérêt à montrer aux élus la variété de l'offre de service.

Pour la majorité des élus (huit sur douze), la qualité du service est aussi assurée par la qualité du fonds documentaire. La bibliothèque est ici décrite comme un lieu qui regorge de trésors. Le fonds documentaire fait donc la richesse de la bibliothèque. Chez les professionnels, le service le plus important rendu aux usagers reste la collection (Smith, 2008). Le public apprécie également particulièrement le fonds documentaire (Public Agenda, 2006). Dans une enquête menée par OCLC (2005), $70 \%$ des personnes interrogées au Canada ont répondu penser prioritairement aux livres quand on évoque la bibliothèque. Les élus municipaux sont donc en phase avec les professionnels et le public lorsqu'ils mettent de lavant la collection comme facteur décisif de la qualité du service de bibliothèque.

La majorité des élus municipaux (sept sur douze) s'entendent pour considérer les espaces comme un élément important de la qualité de la bibliothèque. Si lon tient compte des projets de construction ou d'agrandissement en cours, dix municipalités sur douze sont sensibles au respect de la norme quant aux espaces disponibles par habitant. Généralement, les municipalités appuient donc les bibliothèques en termes de locaux, peut-être parce qu'une construction est une source de fierté pour les élus, une manière de laisser leur trace physiquement dans le paysage municipal, en plus dêtre une attraction pour les citoyens (Shoham \& Yablonka, 2008). Le public est, comme les élus municipaux, particulièrement sensible aux espaces dédiés à la bibliothèque (Ladhari \& Morales, 2008).

Pour la moitié des élus rencontrés, la qualité du service des bibliothèques est assurée par la compétence du personnel. Mais notons que trois d'entre eux ne parlent pas spontanément de cette compétence, qu'ils ne reconnaissent que lorsqu'une question précise à ce sujet leur est posée. Dans les douze cas étudiés et présentés dans le Tableau 2, les bibliothécaires sont généralement à la direction des bibliothèques des grandes villes (trois cas sur quatre) et des villes moyennes (quatre cas sur quatre), tandis que les techniciens en documentation dirigent les bibliothèques des petites villes (trois cas sur quatre). Ceci est logique puisque les directives de l'ASTED (1996) préconisent un bibliothécaire pour 6 ooo habitants. Les responsables de bibliothèques qui ne sont pas issus des milieux documentaires sont plus rares (une grande ville dont l'allocation municipale octroyée à la bibliothèque est inférieure à la moyenne provinciale et une petite ville dont l'allocation municipale octroyée à la bibliothèque est égale ou supérieure à la moyenne provinciale). Notre recherche confirme la pauvreté des bibliothèques en termes de personnel qualifié (Savard, 1998 et 2004), ne serait-ce quau poste de direction. Cela s'explique sans doute par le coût sala- 
rial d'une personne qualifiée et la méconnaissance des qualifications et des compétences respectives des bibliothécaires et des techniciens en documentation de la part des élus, mais aussi plus généralement du public. L'impact positif du personnel, notamment du personnel qualifié, sur la qualité du service reste donc à démontrer aux élus comme au public.

La qualité du service est la valeur prioritaire chez les professionnels (Dole, Hurych \& Koehler, 2000 ; Smith, 2008). Pour les élus, comme pour le public d'ailleurs, la qualité du service passe principalement par les espaces, le fonds documentaire et les activités d'animation. Le personnel et les autres services offerts par la bibliothèque semblent de moindre importance comme facteurs de qualité aux yeux des élus que nous avons rencontrés.

\section{Quand l'élu s'exprime en tant qu'individu}

À travers leurs discours, les élus évoquent également leurs valeurs, leurs convictions personnelles, que sont le goût de la lecture, le souci de la qualité de la langue française et le respect des autres.

\section{Le goût de la lecture}

Sept élus sur douze n'hésitent pas à proclamer leur goût pour la lecture. La lecture permet de "développer la matière grise »; elle est un "stimulant »; elle est synonyme d' " ouverture ». La bibliothèque est décrite comme une "vitrine ", un lieu de découvertes. Le goût de la lecture est une valeur que les élus veulent inculquer aux enfants par l' "éveil à la lecture ». D'ailleurs, le public est lui aussi sensible à cet " amour du livre " (Public Agenda, 2006). Le goût de la lecture est donc une valeur partagée par la majorité des élus et par le public.

\section{Le souci de la langue}

À l'analyse, une valeur est apparue dans un quart des cas, alors quelle nétait jamais mentionnée dans la littérature, à savoir la qualité de la langue. Tous les élus que nous avons rencontrés étaient francophones. Dans leur ville, les communautés francophones et anglophones se côtoient. Ils défendent plutôt l'usage du "bon français » que le bilinguisme.

\section{Le respect}

Quelques rares élus ont évoqué le respect (deux élus), l'accueil de tous (un élu) comme valeurs essentielles des bibliothèques publiques. Également rares sont les élus qui ont parlé du respect de l'individualité (deux élus). Les valeurs humaines et sociales des bibliothèques apparaissent donc de façon très limitée dans le discours des répondants.
Le personnel et les autres services offerts par la bibliothèque semblent de moindre importance comme facteurs de qualité aux yeux des élus que nous avons rencontrés.

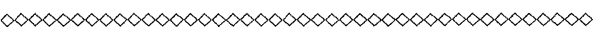

En entrevue, les élus ne sétendent pas longuementsur leurs propres convictions. Aussi, leur discours parait-il limité sur ce point, mais cela ne signifie pas pour autant qu'ils ne sont pas sensibles aux trois éléments précédents.

\section{Quand l'élu s'exprime en tant qu'usager de la bibliothèque}

Les élus que nous avons rencontrés se sont exprimés sur les usages réels ou supposés de la bibliothèque. Ils ont évoqué la bibliothèque comme lieu de rencontre, avec ses publics, ses collections et ses champs d'activité.

\section{La bibliothèque, lieu social}

Pour l'ALA (American Library Association), le rôle de centre d'activités communautaires que devrait jouer la bibliothèque publique invite à développer plusieurs services : des animations, des débats, des rencontres sociales, et l'intégration des communautés culturelles (McClure et al., 1987 ; Hemmel, Wilson \& Revision Committee of the Public Library Association, 1998). Dans le discours des élus municipaux, le rôle de centre d'activités communautaires se traduit par des activités d'animation et des rencontres sociales. La bibliothèque est considérée comme un "lieu de rencontre " par neuf des douze élus.

Lidée de créer des espaces de débat virtuels via la bibliothèque (Chowdhury, Poulter \& McNemeny, 2006) est complètement absente du discours des élus municipaux. Jamais la liberté intellectuelle n’a été évoquée en ces termes. Mais trois élus ont parlé de la bibliothèque en tant que lieu qui permet "la confrontation des idées", "le partage des idées, des conversations " et qui devient "un lieu de dialogue entre les lecteurs ». La bibliothèque est donc un lieu de débat pour un quart des élus municipaux qui ont participé à notre étude. Le public canadien voit également la bibliothèque comme un lieu de débat (Ladhari \& Morales, 2008). Pour plus de la moitié des élus, la bibliothèque joue plutôt le rôle d'une «troisième place " qui permet de rencontrer des personnes en dehors du cercle familial et amical et du cercle professionnel (Lawson, 2004). La bibliothèque est vue comme un espace social (deux tiers des élus) plutôt que civique (un quart des élus). 
Dans le discours des élus, on constate que le public jeune et celui des personnes âgées forment l'essentiel des publics cibles de la bibliothèque.

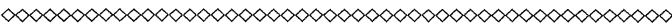

\section{Les publics des bibliothèques}

Les bibliothèques publiques sont ouvertes à tous, mais la littérature présente quelques groupes comme cibles particulières : ce sont principalement les enfants, les adolescents, les personnes âgées, les personnes handicapées et les communautés culturelles. La plupart des élus (neuf élus sur douze) sont particulièrement sensibles aux besoins du public jeune. Ces élus municipaux voient dans la bibliothèque un complément aux milieux scolaires. Les différents âges ne sont pas toujours distincts dans le discours des élus qui parlent de " jeunes " pour désigner autant les enfants dâge préscolaire que les adolescents (trois élus sur douze). Les élus sentendent aussi pour dire que les personnes âgées constituent un public cible des bibliothèques (neuf élus sur douze). Selon eux, les personnes âgées s'ennuient et ont besoin de sortir de chez elles, mais tous les élus ne voient pas la nécessité pour la bibliothèque d'aller au-devant des personnes âgées qui ne peuvent justement pas se déplacer. Les personnes âgées forment donc un public cible reconnu par la plupart des élus municipaux rencontrés, mais la nécessité de mettre en place des services spécifiques pour répondre à leurs besoins est parfois mise en doute. Les nouveaux arrivants constituent un public qui intéresse également quelques élus municipaux des grandes villes (trois élus sur quatre) et des petites villes (deux élus sur quatre), sans qu'ils sachent pour autant comment la bibliothèque pourrait les aider autrement que par l'offre des collections déjà existantes. Seul un élu a parlé du public handicapé, en mentionnant les collections spéciales (pour les personnes malvoyantes notamment) ou les expositions d'œuvres réalisées par des enfants souffrant de déficience intellectuelle. Lorsquon leur pose une question concernant les personnes handicapées, les élus nous parlent des résidences pour personnes âgées ; il y a donc un amalgame dans leur discours entre les personnes âgées et les personnes handicapées. La population handicapée est méconnue et a fortiori ses besoins en matière de bibliothèque.

Dans le discours des élus, on constate que le public jeune et celui des personnes âgées forment l'essentiel des publics cibles de la bibliothèque. D’autres publics, "les plus démunis ", les familles et les analphabètes, ne sont pour leur part que rarement évoqués.

\section{Le respect des ressources mises à la disposition du public}

Le respect des documents na été évoqué en ces termes que par un seul élu. Deux autres ont dit avoir renoncé à la gratuité de l'abonnement de la bibliothèque pour " responsabiliser le citoyen " qui, selon les responsables des bibliothèques, ne prendrait pas soin des documents empruntés quand l'abonnement est gratuit. Le respect est entendu comme respect de l'intégrité physique des documents et non comme respect moral des œuvres; il est donc question de préservation matérielle plutôt que de respect des œuvres de lesprit. La préservation est considérée comme une valeur prioritaire par 10,2 \% des professionnels des bibliothèques publiques (Koehler, Hurych, Dole \& Wall, 2000). Les élus municipaux, comme les professionnels, ne privilégient apparemment pas cette valeur. Deux élus ont parlé de laccès aux documents en faisant référence à des livres "bien classés " dans la bibliothèque, mais lorganisation et le classement ne sont pas des valeurs récurrentes dans le discours des élus rencontrés. Là encore, ces valeurs sont peut-être si évidentes que les élus n'ont pas pensé à en parler lorsque la question des valeurs qui fondent les bibliothèques leur a été posée.

\section{Les champs d'activité des bibliothèques publiques}

Pour l'UNESCO, les champs d'activité des bibliothèques publiques sont l'information, la culture, léducation et lalphabétisation (1994), auxquels nous ajoutons ici le divertissement.

Léducation fait l'unanimité chez les élus : c'est la mission première de la bibliothèque publique selon eux. Dix pour cent seulement des bibliothécaires interrogés par Smith (2008) ont placé la mission éducative de la bibliothèque publique devant les missions d'information, de loisirs, d’animation et autres. Le public, lui, apprécie particulièrement les services offerts à des fins éducatives (Metropolitan Cooperative Library System, 1999 ; Public Agenda, 2006). Si l'éducation est mise de l'avant par les élus, c'est sans doute parce que les répondants accusent les bibliothèques scolaires de désuétude et de pauvreté intenses, mais aussi parce que la fréquentation des bibliothèques publiques par les élèves est reconnue. Or, la fréquentation est un facteur d'influence important aux yeux des élus.

La culture peut être entendue au sens anthropologique du terme ou bien dans le sens de culture générale (Dollot, 1996). La plupart des élus ont évoqué la culture dans ces deux acceptions. Toutefois, il est à remarquer que les élus n'utilisent pas toujours le terme "culture » pour lévoquer. Pour évoquer la culture au sens anthropologique, ils parlent d' "identité », de " miroir de la communauté " ou de "caractère propre». Quelquefois ils précisent : «culture régionale », «culture québécoise ». Les 
élus municipaux rencontrés (neuf sur douze) sont sensibles à la préservation de la culture locale, la culture au sens anthropologique du terme. Pour évoquer la culture au sens de culture générale, ils (onze sur douze) utilisent d'autres termes : " œuvres littéraires", "le monde des idées", "le savoir ", "la culture de base ", "la culture classique ", "les lettres, les arts, et les sciences». La culture est ainsi décrite soit par son contenu disciplinaire (littérature, arts, sciences), soit avec des concepts proches (savoir, idées, connaissances), soit par des qualificatifs (" culture de base ", " culture classique "). La bibliothèque est décrite comme un «foyer culturel », un «pilier culturel » ou encore un "poumon de culture ». La bibliothèque est donc un lieu de vie et de soutien de la culture dans la ville.

La plupart des élus municipaux (dix élus sur douze) évoquent la mission d'information de la bibliothèque publique. Pour eux, l'information se situe dans les documents (imprimés ou électroniques) ; c'est une entité objective. Ils reconnaissent que la bibliothèque permet la recherche d'information. Ceci est également reconnu par les professionnels (Smith, 2008), mais aussi et surtout par le public (ALA, 2006 ; Metropolitan Cooperative Library System, 1999; OCLC, 2005). Pour les professionnels que nous avons rencontrés comme dans la littérature, outre les collections et les moyens d'accès, l'aide à la recherche d'information est essentielle (Sullivan, 2003). Or, du point de vue des élus, la recherche d'information n'implique pas nécessairement l'organisation d'un service à offrir, mais surtout des ressources documentaires à mettre à disposition. Un seul élu a évoqué l'aide aux lecteurs fournie aux usagers qui le demandent; il s'agit du seul cas de bibliothèque dans notre échantillon qui offre un service de référence. Les autres élus ne voient pas la nécessité d'accorder aux lecteurs une aide autre qu'une aide occasionnelle. Ils ne savent pas ce qu'est un service de référence, tandis que la plupart des responsables de bibliothèques rencontrés ont dit souhaiter créer un tel service.

Pour les élus (huit sur douze), le divertissement est assuré par la lecture et par des locaux conviviaux. Dans une enquête menée par l'ALA (2006) auprès du public, $40 \%$ des répondants ont dit aller à la bibliothèque pour se divertir. Les élus sont toujours sensibles aux habitudes et comportements des citoyens, mais le divertissement n'apparait pourtant pas comme une mission prioritaire à leurs yeux. La mission de divertissement ne fait pas l'unanimité chez les élus municipaux, contrairement à ce que pensaient les professionnels québécois (Baillargeon, 2002).

Dans notre échantillon, la moitié des élus municipaux insistent pour que la bibliothèque n’agisse pas seule pour contrer l'analphabétisme, mais travaille de concert avec des partenaires spécialisés. Deux élus ne souhaitent pas que l'alphabétisation incombe aux municipalités, fusse via la bibliothèque. D’autres encore pensent que leur ville n'est pas concernée par ce sujet (trois élus).
Même s'ils utilisent le terme

de "rôle», les élus que nous

avons rencontrés ne conçoivent

pas la bibliothèque comme un

acteur; pour eux, la bibliothèque

est avant tout un lieu.

0000000000000000000000000000

Enfin, pour un autre élu, la bibliothèque contribue à l'alphabétisation, mais l'alphabétisation ne constitue pas pour autant un champ d'activité des bibliothèques. Lalphabétisation nest donc pas prioritaire pour nos élus.

\section{Conclusion 1 : Une vision passive de la bibliothèque}

Pour l'ALA, les rôles de la bibliothèque publique sont au nombre de huit : centre d'activités communautaires, centre d'information communautaire, centre de support éducatif, centre indépendant d’apprentissage, bibliothèque populaire, porte d'apprentissage aux enfants d'âge préscolaire, bibliothèque de référence et centre de recherche (McClure et al., 1987). À chacun de ces rôles correspondent des services à mettre en place (Hemmel, Wilson \& Revision Committee of the Public Library Association, 1998). Même s'ils utilisent le terme de "rôle ", les élus que nous avons rencontrés ne conçoivent pas la bibliothèque comme un acteur; pour eux, la bibliothèque est avant tout un lieu. Les élus reconnaissent les rôles de la bibliothèque publique, mais ne les traduisent pas en services à offrir ou à développer; ils doutent parfois de l'utilité de certains services. Autrement dit, le discours des élus ne présente pas la bibliothèque comme un service, mais plutôt comme un lieu qui met à disposition des ressources, lesquelles peuvent être exploitées de différentes façons. Laction des professionnels est passée sous silence.

\section{Conclusion 2 : Une vision traditionnelle de la bibliothèque}

La mission traditionnelle de la bibliothèque perdure pour les élus, comme pour le public d'ailleurs (Public Agenda, 2006). La mission de la bibliothèque est essentiellement éducative et culturelle. L'information, le divertissement et l'alphabétisation sont également des missions évoquées par une portion des élus rencontrés. Mais, contrairement à ce que nous dit la littérature professionnelle, l'information n'apparait pas comme une mission stratégique dans le discours des élus rencontrés. 


\section{Il s'agit également de baser la stratégie marketing sur les points problématiques dans le discours des élus : la méconnaissance des publics, des services, de la qualité du personnel de la bibliothèque et de la mission d'information.}

\section{Conclusion 3 : Une vision ancrée dans le présent}

La majorité des élus rencontrés (sept sur douze) assure que leur bibliothèque est une réussite. Il ne s'agit donc pas de remettre en cause ce pris pour acquis : tout va bien pour la bibliothèque. Ces élus montrent peu d'incertitudes quant aux bibliothèques publiques si ce nest sur les projets de construction et d'aménagement ou sur les besoins de services particuliers. Sept élus sur douze utilisent le futur ou le conditionnel dans leur discours ; ce sont plutôt des élus des petites villes, sans doute parce qu'ils rêvent d'une bibliothèque plus grande, plus moderne. Ce sont aussi principalement les élus des villes dont l'allocation municipale par habitant octroyée à la bibliothèque est inférieure à la moyenne provinciale. Il s'agit donc des bibliothèques les moins bien dotées; il y a place à amélioration et les élus en sont sans doute conscients. Principalement, les possibilités de développement des bibliothèques publiques concernent des ressources plus importantes, des services élargis pour des publics plus diversifiés et un accès facilité. Quand il est évoqué, lavenir des bibliothèques ne parait donc pas révolutionnaire. Un élargissement des conditions présentes suffit à faire rêver les élus municipaux que nous avons rencontrés. Il semble que nos répondants soient satisfaits de la situation présente et sont, de ce fait, peu enclins à exiger plus.

\section{Conclusion générale}

Notre analyse a montré que les élus que nous avons rencontrés s'intéressent au dossier des bibliothèques publiques, au moins depuis leur élection. Le discours de ces élus est certes limité par rapport à celui des professionnels, ce qui est logique, mais c'est aussi un discours qui converge avec ce discours professionnel, et, surtout, un discours très proche de celui du public. Lanalyse a également soulevé une quadruple problématique en ce qui concerne le point de vue des élus sur les missions des bibliothèques publiques :
- L'élu, qui doit assumer ses responsabilités de gestionnaire de la municipalité, est aussi un individu avec des convictions personnelles et une expérience personnelle par rapport à la bibliothèque. Il offre donc un point de vue construit sur trois voix qui lui sont propres. La problématique est la suivante : le professionnel ne doit pas seulement convaincre l'élu qui détient le pouvoir de décision, mais aussi l'individu et l'usager (réel ou supposé) qu'il est aussi ;

- Les élus ont une vision homogène et tronquée des publics réels et potentiels des bibliothèques publiques. La problématique consiste à changer cette vision basée sur des pris pour acquis et à élargir les perspectives ;

- Les élus ont une image passive de la bibliothèque dans laquelle l'action et la médiation du personnel de la bibliothèque sont invisibles. La problématique réside dans l'évaluation et le marketing des services offerts pour rendre visibles, à la fois, le personnel de la bibliothèque et les services qu'il met en œuvre ;

- Les élus partagent un point de vue traditionnel de la bibliothèque. La problématique est de sensibiliser les élus à la mission d'information de la bibliothèque publique dans la société de l'information dans laquelle nous vivons.

Afin d'améliorer la communication entre les bibliothécaires et les élus municipaux, il s'agit finalement de préconiser un marketing relationnel qui vise à personnaliser le lien entre les bibliothécaires et les élus et à baser cette relation sur les valeurs à partager (c'est-à-dire s'adresser davantage à lélu en tant qu'individu). Il s'agit également de baser la stratégie marketing sur les points problématiques dans le discours des élus : la méconnaissance des publics, des services, de la qualité du personnel de la bibliothèque et de la mission d'information. (-)

\section{Sources consultées}

ALA (American Library Association). 2006. @ your Library:Attitudes toward Public Libraries Survey 2006. <http://www.ala.org/ ala/aboutala/offices/ors/reports/2006KRCReport.pdf>.

ASTED (Association pour l'Avancement des Sciences et des Techniques de la Documentation). 1996. Pour des bibliothèques québécoises de qualité : Guide à l'usage des bibliothèques publiques. Montréal, Québec : ASTED.

Baillargeon, Jean-Paul. 2002. Bibliothèques publiques, gestionnaires et élus municipaux. Documentation et bibliothèques 48 (2): $37-40$.

Bouthillier, France. 1995. Des idéologies et une culture : La signification sociale des bibliothèques publiques. Documentation et bibliothèques 41 (4) : 205-216.

Bouthillier, France. 1996. The Meaning of Service : An Ethnographic Study of a Public Library in Quebec. Thèse de doctorat, Faculty of Information Studies, University of Toronto. Toronto, Ontario. 
1997. Le service et sa signification dans une bibliothèque publique du Québec. Documentation et bibliothèques 43 (3): 115-123.

Chowdhury, Gobinda, Alan Poulter, \& David McMenemy. 2006. At the Sharp End Public Library 2.o : Towards a New Mission for Public Libraries as a " Network of Community Knowledge ». Online Information Review 30 (4) : 454-46o.

Dole, Wanda V., Jikta M. Hurych, \& Wallace C. Koehler. 2000. Values for Librarians in the Information Age : An Expanded Examination. Library Management 21 (6) : 285-297.

Dollot, Louis. 1996. Culture individuelle et culture de masse. $6^{\mathrm{e}}$ éd. Paris : Presses Universitaires de France.

Gomez Hernandez, José. 1998. Legitimación y funciones de la biblioteca en el contexto de la sociedad digital. Scire 4 (2) : 63-77.

Grandmont, Gérald. 2004. Premiers effets de la politique de la lecture et du livre. Dans Bibliothèques publiques et transmission de la culture à l'orée du XXI siècle: Actes du colloque tenu à la bibliothèque Gabrielle-Roy les 5 et 6 mai 2003 à l'occasion du $30^{\circ}$ anniversaire de la fondation de l'ASTED et du $20^{e}$ anniversaire de l'inauguration de la bibliothèque Gabrielle-Roy, sous la direction de Jean-Paul Baillargeon, 89-96. Montréal, Québec : IQRC ; ASTED ; Presses de l'Université Laval.

Hemmel, Ethel, William James Wilson \& Revision Committee of the Public Library Association. 1998. Planning For Results: A Public Library Transformation Process: The Guidebook. Chicago, Illinois : American Public Library Association.

IFLA (International Federation of Library Associations and institutions). 2001. Les services de la bibliothèque publique: Principes directeurs de l'IFLA/UNESCO. URL : <http://www.ifla.org/VII/ s8/news/pgo1-f.pdf>.

Kann-Christensen, Nanna, \& Niels Ole Pors. 2004. The Legitimacy of Public Libraries : Cross-Pressures and Change Processes. New Library World 105 (1204/1205) : 330- 336.

Kerslake, E., \& M. Kinnell. 1998. Public Libraries, Public Interest and the Information Society : Theoretical Issues in the Social Impact of Public Libraries. Journal of Librarianship and Information Science 30 (3) : 159-167.

Koehler, Wallace C., Jikta M. Hurych, Wanda D. Dole, \& Joanna Wall. 2ooo. Ethical Values of Information and Library Professionals : An Expanded Analysis. International Information \& Library Review 32 (3/4) : 485-507.

Ladhari, Riadh, \& Miguel Morales. 2008. Perceived Service Quality, Perceived Value and Recommandation : A Study among Canadian Public Library Users. Library Management 29 (4/5) : 352-366.

Lajeunesse, Marcel. 2005. Le financement des bibliothèques publiques du Québec depuis 1960. Argus 34 (3) : 11-18.

Lawson, Karen. 2004. Libraries in the USA as Traditional and Virtual “Third Places". New Library World 105 (1198/1199) : 125-130.
Lefebvre-Roux, Maud. 1993. Les bibliothèques : Pourquoi pas la fierté de nos élus? Documentation et bibliothèques 39 (4): 213-214.

March, James G., \& Johan P. Olsen. 1976. Ambiguity and Choice in Organizations. Bergen, Norvège : Universitetsforlaget.

McClure, Charles R. et al. 1987. Planning and Role Setting for Public Libraries : A Manual of Options and Procedures. Chicago, Illinois: American Library Association.

McKee, Bob. 1987. Public Libraries - Into the 1990 ? Newcastleunder-Lyme, Royaume-Uni : Association of Assistant Librarians.

Metropolitan Cooperative Library System. 1999. Public Perception of Public Libraries. <http://www.library.ca.gov/lds/docs/METAsummaryo9-99.pdf>.

Muddiman, David. 1990. Towards a Definition of Public Library Purpose. Public Library Journal 5 (4) : 90-94.

OCLC. 2005. Perceptions of Libraries and Information Resources: A Report to the OCLC Membership. <http://www.oclc.org/ reports/pdfs/Percept_all.pdf>.

Poulain, Martine, \& Martine Darrobers. 1992. Les bibliothèques publiques en Europe. Paris : Cercle de la librairie.

Public Agenda. 2006. Long Overdue: A Fresh Look at Public and Leadership Attitudes about Libraries in the 21st Century. $<$ http://www.publicagenda.org/files/pdf/Long_Overdue.pdf $>$.

Rubin, Richard E. 2004. Foundations of Library and Information Science. $2^{\text {nd }}$ ed. New York : Neal-Schuman.

Savard, Réjean. 1998. L'évolution des bibliothèques publiques au Québec de 1960 à 1995. Dans Les bibliothèques québécoises d'hier à aujourd'hui: Actes du colloque de l'ASTED et de l'AQÉI, Trois-Rivières, 27 octobre 1998, sous la direction de Gilles Gallichan \& ASTED, 141-157. Montréal, Québec : ASTED.

2004. Les bibliothèques publiques de 1995 à 2001. Dans État des lieux du livre et des bibliothèques, sous la direction de l'Observatoire de la culture et des communications du Québec, 211-224. <http://www.stat.gouv.qc.ca/observatoire/publicat_obs/ etat_livre.htm\#pdf>.

Shoham, Snunith, \& Israela Yablonka. 2008. Monumental Library Buildings in the Internet Era : The Future of Public Libraries. IFLA Journal 34 (3) : 266-729.

Smith, Susan. 2008. Working Librarians' Perceptions of the Role of the Public Library in the $21^{\text {st }}$ Century. Ph.D. Dissertation, University of Texas at Arlington. Arlington, Texas.

Sullivan, Michael. 2003. The Fragile Future of Public Libraries. Public Libraries 42 (5) : 303-308.

UNESCO. 1994. Manifeste de l'UNESCO sur la bibliothèque publique. <http://www.unesco.org/webworld/libraries/ manifestos/libraman_fr.html $>$. 